\title{
A Review on Various IOT Analytics Techniques for Air Pollution Detection in Fog Computing
}

\author{
Ranjit Kaur \\ M. Tech Scholar \\ Department of Computer Science \& Engineering \\ GNDU Regional Campus, Jalandhar, Punjab, India
}

\author{
Pankaj Deep Kaur, PhD \\ Assistant Professor \\ Department of Computer Science \& Engineering \\ GNDU Regional Campus, Jalandhar, Punjab, India
}

\begin{abstract}
Atmospheric contamination has deteriorated the health of plants and animals all over the globe. In particular, pollutants such as benzene(C6H6) has accelerated the rate of cancer among human beings. Therefore, accurate evaluation of the pollutant in the atmosphere is necessary by traffic supervision in urban areas. To reduce atmospheric contamination, an effective mobile strategy planning must be developed. Currently, the atmospheric contamination is measured using spatially scattered networks with limited sensors. Although, these networks and sensors can evaluate the air pollution accurately, however the sensor expenses and size might limit the operational efficiency. In this paper we discuss about the various techniques of IOT and also discuss the detection of air pollution in fog computing. The overall objective of this paper is to detect the air pollution in IOT and we analyze IOT performs better than other techniques.
\end{abstract}

\section{Keywords}

Foggy or haze images; visibility restoration; air light; dark channel prior.

\section{INTRODUCTION}

The fog Computing paradigm, delineate it has the properties, plus those of system in which facilitates Haze services. This sections requires a close have a look at a couple of essential uses plus providers appealing in which substantiate each of our discussion in preference of the particular Haze since the pure piece of system required for the particular aid for any Internet of Things. Haze computing will be an extremely virtualized platform providing you with calculate, storage space, and networking providers amongst conclude equipment plus traditional Impair Computing Files Centres, normally, yet not only located close to network.

\subsection{Internet Of Things (IOT)}

Internet of Things (IOT) is set being another growing trend following the introduction of World Wide Web itself. Millions and even immeasurable 'wise 'devices are supposed to link to one another as well as trade data and knowledge in the internet. The promoters of the IOT imagine virtually all areas of our existence being paid by these kinds of wise devices. Alarms are common examples of these wise devices. IOT, being a scientific trend, symbolizes not able to connections as well as reach ability. With IOT, 'points 'consider almost any target in the facial skin of the earth, no matter whether this is a speaking machine or a noncommunicating not smart object. Coming from a smart machine to your leaf of your hardwood or a package connected with drinks, anything can participate in Internet. Products develop into speaking nodes via the internet, by means of data connection means, mostly by means of Stereo Rate Identification (RFID) tags.IOT include things like wise products while well. Wise products are the type products which in turn aren't just real organizations, but in addition electronic digital kinds as well as perform little jobs regarding individuals as well as the environment.

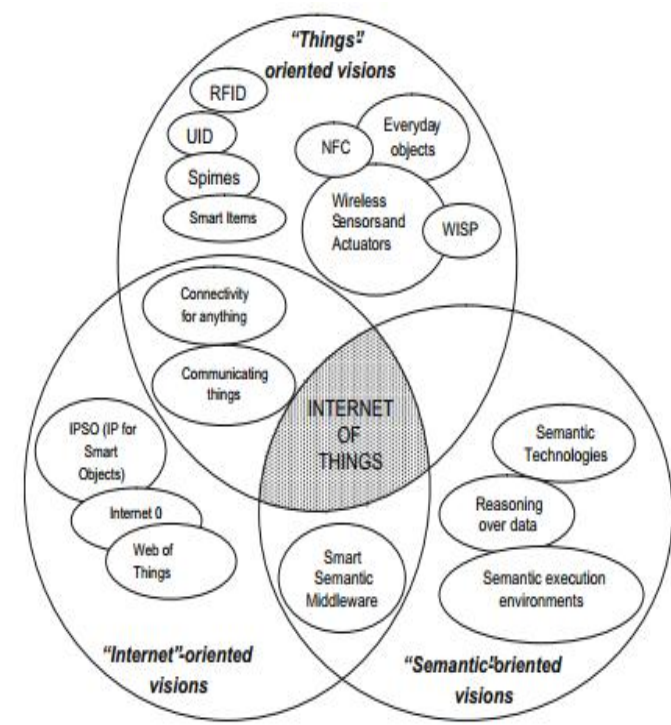

Fig 1: Internet of Things

\subsection{Fog Computing and the Internet of Things}

The actual factor this haze has within about three conditions interesting: Linked Automobile, Clever Power grid, plus Wifi Alarm plus Actuator Networks.

\subsubsection{Connected Vehicle(CV)}

The Connected Automobile deployment displays an excellent case regarding on-line and also affairs: motors in order to motors, motors to gain access to factors (Wi-Fi, 3G, LTE, curbside models [RSUs], sensible targeted traffic lights), and also admittance factors to gain access to points. This Haze has got many qualities which makes it the ideal system to supply an excellent food selection regarding SCV providers around infotainment, security, targeted traffic service, and also analytics: geo-distribution (throughout places and also down roads), flexibility and understanding, minimal latency, heterogeneity, and also service for real-time interactions.

\subsubsection{Smart Grid}

Smart Grid can be another rich Haze utilize case. Haze and also Fog up has gone south Smart Grid.

\subsubsection{Wireless Sensors and Actuators Networks}

The original Wireless Sensor Nodes (WSNs), nicknamed motes, were designed to perform at highly small energy to 
supply battery and also even going to help make vitality cropping feasible. These types of WSNs require many small bandwidth, small vitality, small handling energy, modest memory motes, doing work when reasons for a new drain (collector), in the unidirectional fashion. Smell the planet, straightforward handling, and also sending information towards noise drain would be the duties with these types with indicator cpa networks, is actually the open resource TinyOS2 may be the de-facto conventional doing work system. Motes include demonstrated beneficial in various circumstances to collect environment information (humidity, heat range, degree of rainfall, mild depth, etc)

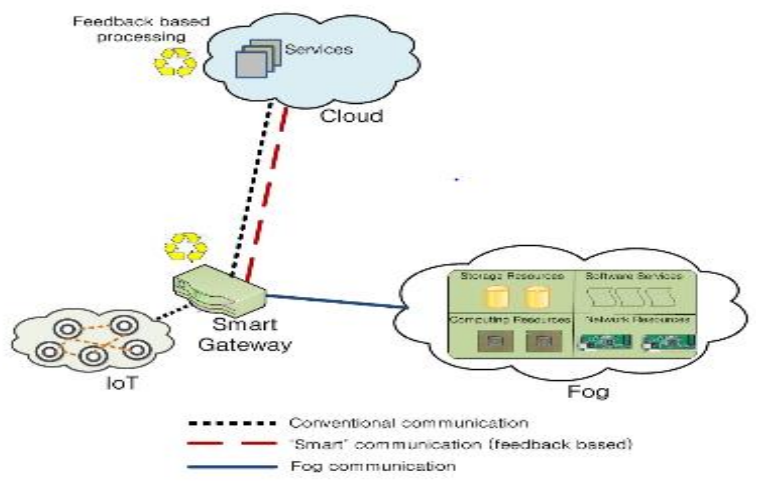

Fig 2: Smart Gateway with Fog computing/Smart network

\section{AIR POLLUTION DETECTION IN FOG COMPUTING}

Pollution is actually unwelcome, detrimental goods contaminating a great environment. This kind to cultivate clean up electricity is actually motivated by high degrees of polluting of the environment that individuals worry are generally once and for all destructive the global environment. Inadequate air quality boosts respiratory illnesses just like bronchial asthma and also respiratory disease, boosts the potential risk of life-threatening problems just like many forms of cancer, and also trouble our health and wellbeing proper care method having important health care costs. Particulate topic is actually singlehandedly responsible for approximately 30,000 quick massive every single year. This instant alterations which the earth is actually watching is actually Worldwide warming[2]. To learn temperature worldwide, boost in seaside levels and also reducing with icecubes via cooler parts and also icebergs, displacement and also decrease of home have ssformerly signaled a great impending tragedy when steps to get maintenance and also normalization aren"et taken on soon. Owning an on-page deadly carbon monoxide propane burglar alarm within your $\mathrm{RV}$, ship, airline or even house essential to mitigate risk. Having the ability to recognize position with deadly carbon monoxide from another location, via when you are, warns you actually to help in a difficulty since it happens and also when chances are you'll be. This makes you actually aware of circumstances prior to deciding to get there, and also means that you can therapy the problem quickly. This cardstock is principally serious in lessening polluting of the environment primarily from your cars making use of IOT.

\section{LITERATURE REVIEW}

Atzori, Luigi et al. [1] addressed the Internet of Things. Principal enabling element with this ensuring paradigm could be the integrated, of countless engineering plus marketing and sales communications solutions. Identity plus checking engineering, sent plus cellular alarm plus actuator systems, elevated communication practices plus handed out thinking ability intended for smart physical objects will be the almost all relevant. Bostock et al. [2] show exactly how representational visibility improves expressiveness and combines using designer methods when compared with preceding approaches, and will be offering equivalent notational efficiency and holding onto highly effective declarative components. Speedy review with workers more shortens debugging and enables iterative development. On top of that, many of us show exactly how D3 converts of course allow computer animation and connection using extraordinary performance enhancements more than more advanced representations.Buyya, Rajkumar et al. [3] defined Reasoning computing and supply the actual structures for building Clouds having market-oriented learning resource permitting by means of benefiting technological know-how for instance Virtual Models (VMs). We also deliver skills with market-based learning resource supervision tactics this cover both customer-driven program supervision and also computational possibility supervision to be able to retain Services Amount Settlement (SLA)-oriented learning resource allocation. On top of that, many of us reveal each of our early on thoughts on interconnecting Clouds for dynamically building global Reasoning exchanges and also markets.Castronova, et al. [4] advances the idea of serviceoriented which by way of representing the layout for your which company of which creates with the Start Geospatial Range (OGC) World-wide-web Producing Service (WPS) protocol. We all exhibit how the WPS protocol could be used to develop which services, and exhibit exactly how most of these which services is usually brought in work-flows surroundings utilizing simple client-side code. We all implemented this method inside the HydroModeler environment, a model coupling software built on the Start Choices in User interface standard. Christin, D et al. [5] expected being built-in to the "Net regarding Things", wherever sensor / probe nodes become a member of a Net dynamically, and employ it to be able to collaborate plus achieve their tasks. However, when WSNs be a part of a Net, we will need to carefully investigate plus examine the issues involved with this integration. With this report, many of us evaluate different methods to combine WSNs to the Net plus format a few issues, which many of us goal to deal with while in the in close proximity to future. Christodoulou, S et al. [6] presented outlines an incorporated method including a final decision service program intended for coming to this sort of "repair-or-replace" selections, during a new long-term pipe advantage operations software which might be carried out by the waters electricity so that you can enhance this longevity of water distribution networks. These advantage operations practices need to conserve the multilevel owners to examine the fitness of water distribution multilevel, evaluate famous event details (leakage or even breakage) in addition to chance of failing, visualise areas of riskly, propose "restoration or even replace" practices in addition to focal points the repair depending on the normal danger and value connected with action.Wong, Brandon P, et al. [7] real-time sensor / probe for potentially have metamorph both equally environment scientific research along with decision-making; this sort of data hardly ever section of real-time workflows, explanations along with custom modeling rendering software chains. Inspite of positive aspects starting from sensing deterioration alerts to flexible sample, your small group along with sophistication connected with current real-time websites all over environment domains present your obstacle towards usage connected with real-time data. Most people provide an architectural mastery developed after 1) your raising variety 
of new technological know-how to reveal environment alerts when website companies, along with 2) your combining connected with these facilities less than latest new developments on the World-wide-web connected with Items (IOT).Diamond, Dermot et al. [8] focused on current trends inside mote-based wi-fi sensor communities and showcased constraints while using recent symptoms these systems, particularly for plug-in of chemo-/biosensing functions and the primary requirement of these products to be completely self-sustaining in most respects if your promised significant scale approximately genuine persistent sensing shall be realized. Díaz, Laura, et al. [9] presented a strategy to increase the signing up regarding alarms plus the publication of their total findings through standards-based Website support interfaces. So that you can make up-to-date plus exact final results, these kinds of products require information with higher temporary plus spatial resolution. Thus, it can be encouraging to take into consideration a raising volume of insitu alarms giving findings of our own natural environment around real-time. We all consider your approach simply by extending a new standards-based GII plus by employing a designed approach for a evidence principle to include in-situ weather conditions findings in to the Euro Natrual enviroment Fireplace Details Program with regard to evaluating hearth hazard around Italy. Gall, Heather E, et al. [10]developed to guide automated selecting regarding hydrographs based on storm-specific information. A vital part of the new "hydrograph-specific selecting scheme" is folks who suffer hydrograph downturn style intended for projecting your hydrograph downturn bend, by which flow-paced time periods will be worked out intended for scheduling the remainder of the samples. The algorithm had been analyzed in the hardwood energy depleted Midwest garden web-site exactly where real-time pass information had been highly processed by way of a programmable information logger in which in turn set off a mechanical sampler on the perfect selecting periods to gather a full regarding thirty products for the duration of just about every weather occurrence independent of the sheer numbers of successive hydrographs made. Gartia, Manas Ranjan et al. [11] presented comprehensive study for the nitrate prognosis outcomes utilizing the miniaturized sensor. We all in addition have exhibited a intergrated, in the sensor to a radio multi-level in addition to completed subject h2o evaluating utilizing the sensor. We all picture that the subject execution in the wi-fi h2o sensor multi-level is going to allow "sensible farming" in addition to "sensible environmentally friendly monitoring".

\section{COMPARISON TABLE}

Table 1. Comparison of various IOT analytic techniques

\begin{tabular}{|c|c|c|c|c|}
\hline Ref no & Authors & Technique & Feature & Limitation \\
\hline 1. & Atzori, Luigi & Internet of Things & $\begin{array}{l}\text { Enhanced communication protocols, } \\
\text { and distributed intelligence for smart } \\
\text { objects are just the most relevant. }\end{array}$ & $\begin{array}{l}\text { The current Internet paradigm, } \\
\text { which supports and has been built } \\
\text { around host-to-host } \\
\text { communications, is now a limiting } \\
\text { factor for the current use of the } \\
\text { Internet. }\end{array}$ \\
\hline 2. & Bostock & $\mathrm{D}^{3}$ Data-Driven & $\begin{array}{l}\text { Representational transparency } \\
\text { improves expressiveness and better } \\
\text { integrates with developer tools than } \\
\text { prior approaches }\end{array}$ & $\begin{array}{l}\text { Evolutionary optimization } \\
\text { techniques have not considered }\end{array}$ \\
\hline 3. & Buyya & Cloud computing & $\begin{array}{l}\text { Encompass both customer-driven } \\
\text { service management and } \\
\text { computational risk management to } \\
\text { sustain Service Level Agreement } \\
\text { (SLA)-oriented resource allocation. }\end{array}$ & $\begin{array}{l}\text { Finally, we need to address } \\
\text { regulatory and legal issues, which } \\
\text { go beyond technical issues. }\end{array}$ \\
\hline 4. & Castronova & $\begin{array}{l}\text { Open Geospatial } \\
\text { Consortium (OGC) }\end{array}$ & $\begin{array}{l}\text { This approach is that the server-side } \\
\text { software follows an established } \\
\text { standard that can be leveraged and } \\
\text { reused within multiple workflow } \\
\text { environments and decision support } \\
\text { systems }\end{array}$ & $\begin{array}{l}\text { Meta-heuristic techniques have not } \\
\text { been considered }\end{array}$ \\
\hline 5. & Christin & $\begin{array}{l}\text { Wireless Sensor } \\
\text { Networks and the } \\
\text { Internet of things }\end{array}$ & $\begin{array}{l}\text { We have selected three important task } \\
\text { assignments in order to highlight the } \\
\text { challenges emerging from the } \\
\text { paradigm adoption: Security, QOS, } \\
\text { and configuration management. }\end{array}$ & $\begin{array}{l}\text { Their analysis revealed that the } \\
\text { solutions currently deployed in the } \\
\text { Internet are not suitable for the } \\
\text { limited sensor node resources and } \\
\text { consequently. }\end{array}$ \\
\hline
\end{tabular}




\begin{tabular}{|l|l|l|l|l|}
\hline 6. & Christodoulou & $\begin{array}{l}\text { Water } \\
\text { Detection }\end{array}$ & $\begin{array}{l}\text { The improvement of the performance } \\
\text { and the reduction of the power } \\
\text { consumption of the network }\end{array}$ & $\begin{array}{l}\text { The risk of failure for } \\
\text { each segment of the pipe network } \\
\text { based on rolling historical data. }\end{array}$ \\
\hline 7. & Brandon P & $\begin{array}{l}\text { Real-time } \\
\text { environmental } \\
\text { sensor data }\end{array}$ & $\begin{array}{l}\text { To make significant gains in the use of } \\
\text { real-time data. }\end{array}$ & $\begin{array}{l}\text { The results are not scalable and } \\
\text { efficient }\end{array}$ \\
\hline 8. & Dermot & $\begin{array}{l}\text { Chemo-/ Bio } \\
\text { sensing }\end{array}$ & $\begin{array}{l}\text { Enhanced communication protocols, } \\
\text { and distributed intelligence for smart } \\
\text { objects are just the most relevant. }\end{array}$ & $\begin{array}{l}\text { Finally, we need to address } \\
\text { regulatory and legal issues, which } \\
\text { go beyond technical issues. }\end{array}$ \\
\hline 9. & Díaz & $\begin{array}{l}\text { Geospatial } \\
\text { Information } \\
\text { Infrastructures }\end{array}$ & $\begin{array}{l}\text { To improve the registration of sensors } \\
\text { and the publication of their } \\
\text { observations via standards-based Web } \\
\text { service interfaces. }\end{array}$ & $\begin{array}{l}\text { Evolutionary } \\
\text { techniques have not considered }\end{array}$ \\
\hline 10. & Heather E & $\begin{array}{l}\text { Hydrograph } \\
\text { modelling with } \\
\text { real-time }\end{array}$ & $\begin{array}{l}\text { The utility of the algorithm was } \\
\text { successfully tested with hydrograph } \\
\text { data collected at both a tile drain and } \\
\text { agricultural ditch, suggesting the } \\
\text { potential for general applicability of } \\
\text { the method }\end{array}$ & $\begin{array}{l}\text { The current Internet paradigm, } \\
\text { which supports and has been built } \\
\text { around } \\
\text { communications, is now a limiting } \\
\text { factor for the current use of the } \\
\text { Internet. }\end{array}$ \\
\hline
\end{tabular}

\section{CONCLUSION}

IOT integrates various devices equipped with sensing, identification, processing, communication, and networking capabilities. In particular, sensors and actuators are getting increasingly powerful, less expensiveand smaller, which makes their use ubiquitous.This paper represents the comparison between various IOT techniques by which we detect the air pollution in fog computing. The main Objective of this paper is to predict the benzene quantity in atmosphere using various regression techniques in order to accurately monitor the air pollution. When sensor fusion techniques are utilized in interpolation methods, they must be tuned appropriately via training by using reference information. In this, different well-known regression algorithms are considered. Their improvement is done by using Adaptive Neural Fuzzy Inference System on FOG computing.

\section{REFERENCES}

[1] Atzori, Luigi, Antonio Iera, and Giacomo Morabito. 2010The internet of things: A survey.

[2] Bostock, Michael, Vadim Ogievetsky, and Jeffrey Heer. $2011 D^{3}$ data-driven documents. IEEE transactions on visualization and computer graphics.

[3] Buyya, Rajkumar, et al. 2009Cloud computing and emerging IT platforms: Vision, hype, and reality for delivering computing as the 5th utility.

[4] Castronova, Anthony M., Jonathan L. Goodall, and Mostafa M. Elag.2013 Models as web services using the open geospatial consortium $(\mathrm{ogc})$ web processing service (wps) standard.

[5] Christin, D., Reinhardt, A., Mogre, P.S., Steinmetz, R. 2009 Wireless sensor networks and the internet of things: selected challenges.
[6] Christodoulou, S., et al. 2010Wireless sensor networks for water loss detection.

[7] Wong, Brandon P., and Branko Kerkez. 2016Real-time environmental sensor data: An application to water quality using web services.

[8] Diamond, Dermot, et al. 2008 Wireless sensor networks and chemo-/biosensing.

[9] Díaz, Laura, et al. 2013 Publishing sensor observations into Geospatial Information Infrastructures: A use case in fire danger assessment. Environmental modelling \& software.

[10] Gall, Heather E., Chad T. Jafvert, and Byron Jenkinson. 2010 Integrating hydrograph modeling with real-time flow monitoring to generate hydrograph-specific sampling schemes.Journal of hydrology .

[11] Gartia, Manas Ranjan, et al. 2012 The microelectronic wireless nitrate sensor network for environmental water monitoring. Journal of Environmental Monitoring.

[12] Gronewold, Andrew D., et al. 2013 A dynamic graphical interface for visualizing projected, measured, and reconstructed surface water elevations on the earth's largest lakes. Environmental modelling \& software.

[13] Gubbi, Jayavardhana, et al. 2013 Internet of Things (IoT): A vision, architectural elements, and future directions.Future generation computer systems.

[14] Jin, Ning, et al. 2010 A novel design of water environment monitoring system based on wsn. Computer Design and Applications (ICCDA). 